\title{
Mechanically driven spin-orbit-active weak links
}

\author{
M. Jonson and R.I. Shekhter \\ Department of Physics, University of Gothenburg, SE-41296 Göteborg, Sweden \\ O. Entin-Wohlman and A. Aharony \\ Raymond and Beverly Sackler School of Physics and Astronomy, Tel Aviv University, Tel Aviv 69978, Israel \\ Physics Department, Ben Gurion University, Beer Sheva 84105, Israel \\ H.C. Park \\ Center for Theoretical Physics of Complex Systems, Institute for Basic Science (IBS) \\ Daejeon 34051, Republic of Korea \\ D. Radić \\ Department of Physics, Faculty of Science, University of Zagreb, Bijenicka 32, Zagreb 10000, Croatia \\ E-mail: dradic@phy.hr
}

Received August 30, 2018, published online October 26, 2018

\begin{abstract}
We show that new functionality of spin-orbit-active electronic weak links can be achieved by their timedependent mechanical deformation. As an illustration we use a simple model to calculate the electronic spin current generated by rotating a bent spin-orbit-active nanowire coupled to bulk metallic leads.
\end{abstract}

Keywords: spin-active weak links, electronic spin current.

An electric weak link in the form of a nanoconductor, bridging two bulk reservoirs of electrons, is a mesoscopic device where electronic charge and spin may be accumulated and controlled electrically and magnetically. In this context, spin-active weak links made of materials with strong spin-orbit (SO) interaction [1,2] have recently been of special interest. Splitting electrons transferred through such a weak link with respect to their spin polarization (Rashba splitting) [3] can, e.g., be employed for generating a number of interference- and spin-relaxation phenomena, which bring new spintronic functionality to such nanodevices [4].

Spin-orbit interactions can be induced by external or internal (crystal) electric fields. Here we focus on the latter case and imagine the weak link to be formed by a singlewall carbon nanotube, in which crystal fields caused by elastic strains can be thought of as occurring when a flat graphene ribbon is rolled up to form a tube. The straininduced SO coupling in a one-dimensional model of such a nanotube can be described by the Hamiltonian [4]

$$
\mathcal{H}_{\text {so }}=\hbar v_{F} k_{\text {so }} \sigma \cdot \hat{s} \text {, }
$$

which is a simplified form of the SO Hamiltonian derived for a more realistic nanotube model [5]. In Eq. (1) $v_{F}$ is the Fermi velocity, $k_{s o}$ is a phenomenological parameter that gives the strength of the SO interaction in units of inverse length, $\sigma=\left(\sigma_{x}, \sigma_{y}, \sigma_{y}\right)$ is a vector whose components are the Pauli spin matrices, and $\hat{\mathbf{s}}$ is a unit vector pointing along the longitudinal axis of the nanotube.

In semiclassical terms the effect of the SO interaction can be understood as a precession of the electron's spin around an effective magnetic field $\mathbf{B}_{s o}$, where if the $\mathrm{SO}$ interaction is given by Eq. (1), $\mathbf{B}_{s o}=-\left(\hbar v_{F} k_{s o} / \mu_{B}\right) \hat{s}$. Quantum mechanically, its effect can be accounted for by adding an extra phase, $\varphi_{\mathrm{AC}}$, to its wave function when an electron propagates through an SO-active spatial domain. This is known as the Aharonov-Casher effect, [6] and the Aharonov-Casher phase $\varphi_{\text {AC }}$ can significantly modify electron transport.

The effect of the SO interaction we have in mind can be illustrated by considering the device sketched in Fig. 1, which one can think of as an SO-active electric weak link. Here a (1D) wire (a simple model of a carbon nanotube) serves as a tunneling weak link between two bulk nonmag- 


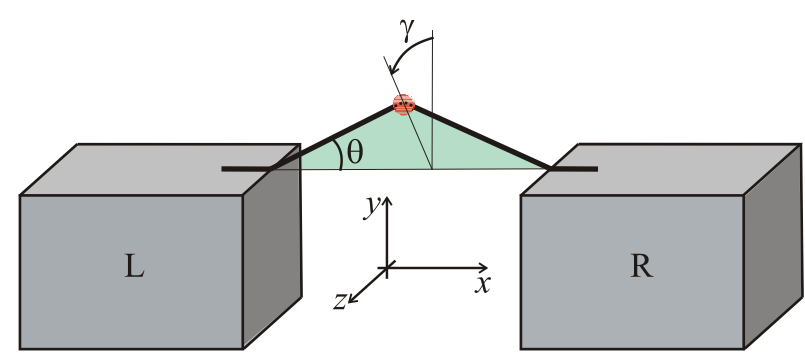

Fig. 1. Illustration of the geometry used to calculate the spin-orbit coupling dependence of the tunneling amplitude. Two straight segments, modeling the bent nanowire (bending is parameterized by the angle $\theta$ ), are tunnel-coupled to left $(\mathrm{L})$ and right $(\mathrm{R})$ electronic electrodes. The spatial orientation of the wire is controlled by the angle $\gamma$ between the $y$-axis and the plane containing the bent nanowire. The setup corresponds to a configuration in which the wire is controlled only mechanically.

netic conductors. A strong spin-orbit interaction is assumed to be present in the wire (but not in the bulk conductors), so that tunneling transfer of electrons through the wire is accompanied by spin-precession dynamics and consequently by the accumulation of an extra phase factor, containing the Aharonov-Casher phase $\varphi_{\mathrm{AC}}$, in the definition of tunneling probability amplitudes,

$$
V_{\mathbf{k p}}=V_{0} \exp \left(i \varphi_{\mathrm{AC}}\right) .
$$

Here we have introduced the tunneling amplitude $V_{0}$ for the left-to-right lead transitions in the absence of the SO interaction [7].

In the proposed device the wire is supposed to be suspended and mechanically bent. The bent wire is modeled as two non-collinear straight segments of equal length $d / 2$, where the angle $\theta$ shown in Fig. 1 describes their degree of direction mismatch. For this geometry the AharonovCasher phase factors were calculated in Ref. 3 for the case when the angle $\gamma$ between the plane containing the bent wire and the $y$-axis (see Fig. 1) is zero. A straightforward generalization yields the result

$$
\exp \left(i \varphi_{\mathrm{AC}}\right)=A+i \mathbf{B} \cdot \boldsymbol{\sigma}
$$

where

$$
\begin{gathered}
A=\cos ^{2}(\alpha)-\sin ^{2}(\alpha) \cos (2 \theta) ; \\
\mathbf{B}=(\sin (2 \alpha) \cos (\theta), \\
\sin (\gamma) \sin ^{2}(\alpha) \sin (2 \theta), \\
\left.-\cos (\gamma) \sin ^{2}(\alpha) \sin (2 \theta)\right)
\end{gathered}
$$

and the dimensionless parameter $\alpha \equiv k_{\text {so }} d / 2$ is proportional to the SO interaction strength.

The Hamiltonian of the system is

$$
\mathcal{H}=\mathcal{H}_{\text {link }}+\mathcal{H}_{\text {leads }}+\mathcal{H}_{\text {tun }} \cdot
$$

Here the Hamiltonian describing the weak link is

$$
\mathcal{H}_{\text {link }}=-\frac{\hbar^{2}}{2 m^{*}} \nabla^{2}+\hbar v_{F} k_{\text {so }} \sigma \cdot \hat{s} \text {. }
$$

The Hamiltonian of the leads is $H_{\text {leads }}=\sum_{\alpha=L, R} \mathcal{H}_{\text {lead }}^{\alpha}$, with

$$
\mathcal{H}_{\text {lead }}^{\alpha=L(R)}=\sum_{\mathbf{k}(\mathbf{p}), \sigma} \varepsilon_{k(p)} C_{\mathbf{k}(\mathbf{p}) \sigma}^{\dagger} \begin{array}{r}
\alpha=L, R \\
C_{\mathbf{k}(\mathbf{p}) \sigma},
\end{array}
$$

where $c_{\mathbf{k} \sigma}^{\dagger}\left(c_{\mathbf{k} \sigma}\right)$ creates (annihilates) an electron with momentum $\mathbf{k}$ and spin $\sigma$ in the left lead (L), with similar definitions in the right lead (R) $[\sigma= \pm$, corresponding to spin projections $\uparrow, \downarrow$ on the spin quantization axis, which we take to be the $z$-axis]. Tunneling between the leads and the weak link is described by

$$
\mathcal{H}_{\text {tun }}=\sum_{\mathbf{k}, \mathbf{p}, \sigma, \sigma^{\prime}}\left(\left[V_{\mathbf{k p}}\right]_{\sigma \sigma^{\prime}} C_{\mathbf{k} \sigma}^{\dagger} c_{\mathbf{p} \sigma^{\prime}}+\left[V_{\mathbf{k p}}^{*}\right]_{\sigma \sigma^{\prime}} C_{\mathbf{p} \sigma^{\prime}}^{\dagger} c_{\mathbf{k} \sigma}\right),
$$

where $\left[V_{\mathbf{k p}}\right]_{\sigma \sigma^{\prime}}$, being the matrix element of the operator (3), is the tunneling amplitude from the state with momentum $\mathbf{p}$ and spin $\sigma^{\prime}$ in the right lead to the state with momentum $\mathbf{k}$ and spin $\sigma$ in the left one.

Following the standard approach we treat the tunneling Hamiltonian perturbatively describing the electronic systems in both leads in the absence of tunneling, each being in local equilibrium, having different chemical potentials, $\mu_{L}$ and $\mu_{R}$, for different leads. Then, the distribution function of electrons in each $(L, R)$ lead is the Fermi function

$$
f_{L, R}\left(\varepsilon_{k}\right)=1 /\left[\mathrm{e}^{\beta\left(\varepsilon_{k}-\mu_{L, R}\right)}+1\right],
$$

where $\beta \equiv 1 / k_{B} T$ is inversely proportional to the temperature $T$. It is essential to note that we consider a nonmagnetic weak link, so the electron energies in the left (right) lead $\varepsilon_{k(p)}$ do not depend on the electron spin.

Charge and spin currents injected through the weak link into each lead are given by the standard expressions

$$
\begin{aligned}
& I_{\text {charge }}^{(L, R)}=\sum_{\sigma} e \frac{d}{d t}\left\langle\sum_{\mathbf{k}(\mathbf{p})} c_{\mathbf{k}(\mathbf{p}) \sigma}^{\dagger} c_{\mathbf{k}(\mathbf{p}) \sigma}\right\rangle_{(L, R)}, \\
& I_{\mathrm{spin}}^{(L, R)}=\sum_{\sigma} \sigma \frac{d}{d t}\left\langle\sum_{\mathbf{k}(\mathbf{p})} c_{\mathbf{k}(\mathbf{p}) \sigma}^{\dagger} C_{\mathbf{k}(\mathbf{p}) \sigma}\right\rangle_{(L, R)} .
\end{aligned}
$$

Note that the spin current $I_{\mathrm{spin}}^{L(R)}$ is given by the time derivative of the $z$-component of the magnetization in the left (right) lead. This is why Eq. (11) represents the flow of the $z$-component of the electronic spin injected into the left (right) lead. For brevity we will simply refer to $I_{\text {spin }}^{L(R)}$ as the "spin current" in what follows. Here, the quantum averages $\langle\ldots\rangle_{L, R}$ are taken over the electronic states in the $(L, R)$ lead, which is assumed to be in local equilibrium. As we can see from Eqs. (2), (3) and (8), the tunneling of an electron through the weak link is coupled to the possi- 
bility of flipping its spin. Therefore such a tunneling event may lead to additional spin production, which is why one may expect that the spin-active electric weak link presented in Fig. 1 can act as a source of spin that can be injected into the conducting leads to create spin currents there. However, a direct calculation of the spin current (11) using Eq. (8) shows that no net spin current is generated by the weak link. Moreover, one can show that no effect of SO coupling on transport is possible for the given setup. The fundamental reason lies in the time-reversal symmetry of the Hamiltonian (5) [8]. Therefore, time-reversal symmetry should be broken in order to activate the effect of Rashba dynamics on electronic transport. Since there is no proof that breaking time-reversal symmetry would accomplish this task irrespective of how the symmetry is broken, one needs to prove it in each special case. For example, it was shown in Ref. 9 that breaking time-reversal symmetry by switching on an external magnetic field, indeed activates spin production in the spin-active weak link of Fig. 1. In this case the possibility to control the induced spin currents electrically and magnetically was also demonstrated.

In this paper we explore the possibility to activate spin production in the weak link by breaking time-reversal symmetry in a different way, viz. via a time-dependent spin-orbit coupling. A number of ways to achieve a timedependent SO interaction can be suggested. Microwave actuation, which exploits the time-dependent electric field responsible for the spin-orbit coupling, is one possible way. Mechanical actuation, which can be achieved either by flexural vibrations of the nanowire or by rotating the bent nanowire around the $x$-axis (see Fig. 1), is another. Below we consider the effect of a rotational activation of the device. We will prove that such mechanical dynamics indeed leads to the generation of a spin current and demonstrate the possibility to manipulate this current electrically and mechanically.

In order to study rotational activation of the nanowire weak link we let the angle $\gamma$ between the plane that contains the bent nanowire and the $y$-axis (see Fig. 1) be a linear function of time,

$$
\gamma=\Omega t
$$

so that $\Omega$ is the rotation frequency of the wire. A generalization of the approach outlined above for the static nanowire weak-link geometry to the case of a rotating wire requires an analysis of electron tunneling through a mechanically driven device. The result should depend on the ratio between the rotation period $\sim 1 / \Omega$ of the wire and the time $\tau_{d} \sim d / v_{F}$ it takes for an electron to pass through the device. We will consider the limiting case when $\Omega \tau_{d} \ll 1$ for which we approximate the Hamiltonian for the rotating-wire problem at hand with the Hamiltonian for the static-wire problem as described by Eqs. (2)-(8) but modified by replacing $\gamma$ by $\gamma(t)$ in Eq. (4) with $\gamma(t)$ as given by Eq. (12) and $t$ treated as a parameter. We shall emphasize this change by using the notation

$$
\mathbf{B}(t) \equiv \mathbf{B}(\gamma(t)) .
$$

Following the standard procedure, one gets to second order in the electronic tunneling rates

$$
\begin{aligned}
& I_{\sigma}^{L}=-2 i \int_{t}^{\infty} d t^{\prime} \sum_{k, p} f\left(\varepsilon_{p}\right)\left[1-f\left(\varepsilon_{k}\right)\right] \times \\
& \times \sin \left(\left(\varepsilon_{k}-\varepsilon_{p}\right)\left(t^{\prime}-t\right)\right)\left[X\left(t, t^{\prime}\right)\right]_{\sigma \sigma},
\end{aligned}
$$

where

$$
\begin{aligned}
X\left(t, t^{\prime}\right) & =\left|V_{0}\right|^{2}\left((A-i \mathbf{B}(t) \cdot \boldsymbol{\sigma})\left(A+i \mathbf{B}\left(t^{\prime}\right) \cdot \boldsymbol{\sigma}\right)\right)- \\
& \left.-\left(A-i \mathbf{B}\left(t^{\prime}\right) \cdot \boldsymbol{\sigma}\right)(A+i \mathbf{B}(t) \cdot \boldsymbol{\sigma})\right) .
\end{aligned}
$$

Further simplifications follow from the properties of the Pauli matrices, which allow us to write the diagonal elements of the operator $X\left(t, t^{\prime}\right)$ as

$$
\begin{gathered}
{\left[X\left(t, t^{\prime}\right)\right]_{\sigma \sigma}=i \sigma\left\{P(\alpha, \theta)\left[\cos \left(\Omega t^{\prime}\right)-\cos (\Omega t)\right]+\right.} \\
\left.+Q(\alpha, \theta)\left[\sin \left(\Omega t^{\prime}\right)-\sin (\Omega t)\right]\right\}
\end{gathered}
$$

where

$$
\begin{aligned}
& P(\alpha, \theta)=2\left[\sin ^{2}(\alpha) \cos (2 \theta)-\cos ^{2}(\alpha)\right] \sin ^{2}(\alpha) \sin (2 \theta), \\
& Q(\alpha, \theta)=2 \sin (2 \alpha) \cos \theta \sin ^{2}(\alpha) \sin (2 \theta) .
\end{aligned}
$$

The integration over time in Eq. (14), using expressions (16) and (17), can be performed analytically, yielding the following expression for the flux of electrons with spin $\sigma$,

$$
\begin{gathered}
I_{\sigma}^{L}=\pi \sigma v^{2}\left|V_{0}\right|^{2} \Omega\{-P(\alpha, \theta) \sin (\Omega t)+Q(\alpha, \theta) \cos (\Omega t)\}= \\
=2 \pi \sigma v^{2}\left|V_{0}\right|^{2} \sin ^{2}(\alpha) \sin (2 \theta) \Omega\{\cos (\theta) \cos (\Omega t)- \\
\left.\quad-\left[\sin ^{2}(\alpha) \cos (2 \theta)-\cos ^{2}(\alpha)\right] \sin (\Omega t)\right\}
\end{gathered}
$$

Here $v$ is the electron density of states, which is assumed to be the same in both leads. From the result (18) we can get the charge current in an electrically unbiased device $\left(\mu_{L}=\mu_{R}\right)$ as

$$
I_{\text {charge }}^{L}=e \sum_{\sigma= \pm} I_{L \sigma}=0
$$

and the spin current (11) as

$$
\begin{gathered}
I_{\text {spin }}^{L}=\sum_{\sigma= \pm} \sigma I_{L \sigma}= \\
=4 \pi v^{2}\left|V_{0}\right|^{2} \sin ^{2}(\alpha) \sin (2 \theta) \Omega\{\cos (\theta) \cos (\Omega t)- \\
\left.-\left[\sin ^{2}(\alpha) \cos (2 \theta)-\cos ^{2}(\alpha)\right] \sin (\Omega t)\right\} .
\end{gathered}
$$


As expected, the charge current is zero for an electrically unbiased device. Also, the spin current is zero under the following conditions: (i) if there is no spin-orbit interaction ( $\alpha=0)$; (ii) if the wire is not bent $(\theta=0)$, or (iii) if it is "bent back on itself" $(\theta=\pi / 2)$; (iv) if the wire is not rotating $(\Omega=0)$. Apart from that, the spin current (more precisely the flow of the $z$-component of the electronic spin) is purely oscillatory (no dc component) with an amplitude that is proportional to the rotation frequency $\Omega$, being neither an even nor an odd function of $\Omega$.

A convenient way to express the spin current is in terms of an amplitude and phase shift of an oscillatory function,

$$
I_{\text {spin }}(t)=I_{\Omega}(\alpha, \theta) \sin \left(\Omega t+\gamma_{0}\right),
$$

where amplitude and phase shift are given by

$$
\begin{gathered}
I_{\Omega}(\alpha, \theta)=\Omega \frac{G}{G_{0}} \sin ^{2}(\alpha) \sin (2 \theta) \times \\
\times \sqrt{\cos ^{2}(\theta)+\left(\cos ^{2}(\alpha)-\sin ^{2}(\alpha) \cos (2 \theta)\right)^{2}}, \\
\gamma_{0}=\arctan \frac{\cos (\theta)}{\cos ^{2}(\alpha)-\sin ^{2}(\alpha) \cos (2 \theta)} .
\end{gathered}
$$

Here, we expressed the amplitude $I_{\Omega}(\alpha, \theta)$ in terms of the conductance $G$, using the identity $4 \pi v^{2}\left|V_{0}\right|^{2}=G / G_{0}$, where $G_{0}=e^{2} / h$ is the conductance quantum. The dependence of $I_{\Omega}$ and $\gamma_{0}$ on $\alpha$ and $\theta$ is illustrated in Fig. 2.

With respect to the physics behind the phenomenon of spin generation, there is an illuminating analogy between our problem and the process of spin-flip-assisted electronic microwave absorption [10]. The interaction between the spin of an electron and the time-dependent magnetic component of a microwave electro-magnetic field has the same form as the spin-orbit coupling, which couples the spin to the effective time-dependent "effective" magnetic field $\mathbf{B}_{\text {so }}$ introduced in the introduction to this paper. A circular po-

(a)

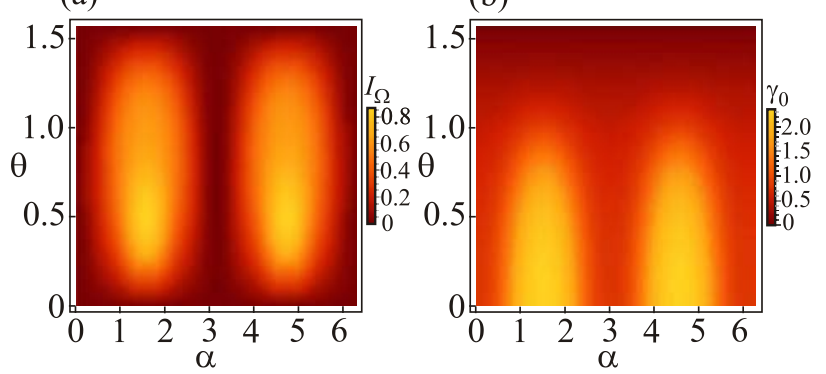

Fig. 2. (Color online) Graphical representation of the dependence of the temporally oscillating spin current (21) on the dimensionless strength $\alpha$ of the SO interaction and the bending angle $\theta$ of the wire: (a) Amplitude of the spin current $I_{\Omega}(\alpha, \theta)$ measured in units of $\Omega G / G_{0}$ as given by Eq. (22). (b) Phase shift of the spin current $\gamma_{0}(\alpha, \theta)$ as given by Eq. (23). larization of the microwave field, providing a time-varying orientation of its magnetic component, induces spin-flipassisted electronic transitions involving absorption or emission of microwave photons. In our case, the rotation of the bent nanowire weak link changes the spatial orientation of $\mathbf{B}_{\text {so }}$ in time, thus bringing a certain "circularity" to the effective "spin-orbit microwave field" and consequently inducing an asymmetry between the flipping up or down of a spin during an emission or absorption event. In our device, at zero temperature, only absorption transitions are possible due to the Pauli constraint. In a further analogy with the microwave case, one can think of momentum being conserved in such an absorption event by the transfer of a finite amount of the rotational momentum of the weak link to the precessing spin, a transfer facilitated in our case by the time-dependent spin-orbit interaction.

From expression (21) one can easily see that the maximal spin, accumulated during one half-period of timeoscillation

$$
S=\frac{1}{\Omega} \int_{0}^{\pi} I_{\text {spin }}(\tau) d \tau,
$$

where $\tau \equiv \Omega t+\gamma_{0}$, equals $S=2 I_{\Omega}(\alpha, \theta)$. The value of $S$ can be estimated using Eq. (22). For a wire of length $d \sim 1$ $\mu \mathrm{m}$ and an SO interaction strength corresponding to $k_{s o} \sim 0.2(\mu \mathrm{m})^{-1}$, a value, which can be extracted from measured energy splittings caused by strain-induced SO interactions in single-walled carbon nanotubes [11], one finds that $\alpha \sim 1$. This allows us to estimate $S$ to be of the order of the spin of a single electron.

\section{Acknowledgment}

This work was supported by the Institute for Basic Science in Korea (IBS-R024-D1); the Swedish Research Council (VR); the Israel Science Foundation (ISF), by the infrastructure program of Israel Ministry of Science and Technology under contract 3-11173, and by the Pazy Foundation; the Croatian Science Foundation, project IP2016-06-2289, and by the QuantiXLie Centre of Excellence, a project cofinanced by the Croatian Government and the European Union through the European Regional Development Fund - the Competitiveness and Cohesion Operational Programme (Grant KK.01.1.1.01.0004).

1. G. Dresselhaus, Phys. Rev. 100, 580 (1955).

2. E.I. Rashba and V.I. Sheka, Fiz. Tverd. Tela: Collected Papers 2, 162 (1959).

3. R.I. Shekhter, O. Entin-Wohlman, and A. Aharony, Phys. Rev. Lett. 111, 176602 (2013); R.I. Shekhter, O. EntinWohlman, and A. Aharony, Phys. Rev. B 90, 045401 (2014).

4. R.I. Shekhter, O. Entin-Wohlman, M. Jonson, and A. Aharony, Fiz. Nizk. Temp. 43, 368 (2017) [Low Temp. Phys. 43, 303 (2017)]. 
5. M.S. Rudner and E.I. Rashba, Phys. Rev. B 81, 125426 (2010); K. Flensberg and C.M. Marcus, Phys. Rev. B 81, 195418 (2010).

6. Y. Aharonov and A. Casher, Phys. Rev. Lett. 53, 319 (1984).

7. For simplicity we neglect the (k, p)-momentum dependence of the tunneling amplitude, where $\mathbf{k}(\mathbf{p})$ denote momenta of electronic states in left (right) lead.

8. J.H. Bardarson, J. Phys. A: Math. Theor. 41, 405203 (2008).

9. A. Aharony, O. Entin-Wohlman, M. Jonson, and R.I. Shekhter, Phys. Rev. B 97, 220404(R) (2018).

10. F. Meier and B.P. Zakharchenya (eds.), Optical Orientation, §7 Elsevier Science Publishers B. V., North-Holland, Amsterdam (1984).

11. F. Kuemmeth, S. Ilani, D.C. Ralph, and P.L. McEuen, Nature 452, 448 (2008).

\section{Механічно керовані слабкі зв'язки зі спін-орбі- тальною взаємодією}

\section{Jonson, R.I. Shekhter, O. Entin-Wohlman, A. Aharony, H.C. Park, D. Radić}

Обговорюються нові функціональні можливості, що реалізовуються у тунельних структурах завдяки залежній від часу сильній спін-орбітальній взаємодії електронів. Як приклад запропоновано просту модель, в якій передбачено генерацію спінового струму, що виникає при обертанні зігнутого дроту, який з’єднує два масивні метали, із локалізованою на ній сильною спін-орбітальною взаємодією електронів.

Ключові слова: спін-активні слабкі зв’язки, електронний спіновий струм.

\section{Механически управляемые слабые связи со спин- орбитальным взаимодействием}

\author{
M. Jonson, R.I. Shekhter, O. Entin-Wohlman, \\ A. Aharony, H.C. Park, D. Radić
}

Обсуждаются новые функциональные возможности, реализующиеся в туннельных структурах благодаря зависящему от времени сильному спин-орбитальному взаимодействию электронов. В качестве примера предложена простая модель, в которой предсказана генерация спинового тока, возникающая при вращении изогнутой проволоки, соединяющей два массивных металла, с локализованным на ней сильным спинорбитальным взаимодействием электронов.

Ключевые слова: спин-активные слабые связи, электронный спиновый ток. 